\title{
Correction to: A Lattice Based Algebraic Model for Verb Centered Constructions
}

\author{
Bálint Sass
}

\author{
Correction to: \\ Chapter "A Lattice Based Algebraic Model for Verb \\ Centered Constructions" in: P. Sojka et al. (eds.): \\ Text, Speech, and Dialogue, LNAI 11107, \\ https://doi.org/10.1007/978-3-030-00794-2_25
}

In the paper titled "A Lattice Based Algebraic Model for Verb Centered Constructions", an acknowledgement has been added. 